\title{
A Cytotoxic Indole Characterized by Incorporation of a Unique Carbon- Nitrogen Skeleton and Two Pentacyclic Corynanthean Alkaloids Incorporating a Substituted Tetrahydrofuranone Ring from Kopsia arborea
}

Soon-Kit Wong, ${ }^{\dagger}$ Suet-Pick Wong, ${ }^{\dagger}$ Kae-Shin Sim, ${ }^{\dagger}$ Siew-Huah Lim,${ }^{\dagger}$ Yun-Yee Low $, *, \dagger$ and Toh-Seok Kam*,†

${ }^{\dagger}$ Department of Chemistry, Faculty of Science, University of Malaya, 50603 Kuala Lumpur, Malaysia

${ }^{\ddagger}$ Institute of Biological Sciences, Faculty of Science, University of Malaya, 50603 Kuala Lumpur, Malaysia. 


\section{Table of Contents}

No.

Page

$1 \quad$ Figure $\mathrm{S} 1 .{ }^{1} \mathrm{H} \mathrm{NMR}\left(\mathrm{CDCl}_{3}, 400 \mathrm{MHz}\right)$ spectrum of arbophyllidine (1) 3

2 Figure $\mathrm{S} 2 .{ }^{13} \mathrm{C} \mathrm{NMR}\left(\mathrm{CDCl}_{3}, 100 \mathrm{MHz}\right)$ spectrum of arbophyllidine (1) 4

3 Figure $\mathrm{S} 3 .{ }^{1} \mathrm{H} \mathrm{NMR}\left(\mathrm{CDCl}_{3}, 600 \mathrm{MHz}\right)$ spectrum of arbophyllinine $\mathrm{A}(2)$

4 Figure $\mathrm{S} 4 .{ }^{13} \mathrm{C}$ NMR $\left(\mathrm{CDCl}_{3}, 150 \mathrm{MHz}\right)$ spectrum of arbophyllinine $\mathrm{A}(\mathbf{2})$

5 Figure S5. ${ }^{1} \mathrm{H}$ NMR (Methanol- $d_{4}, 600 \mathrm{MHz}$ ) spectrum of arbophyllinine B (3) 7

6 Figure S6. ${ }^{13} \mathrm{C}$ NMR (Methanol- $d_{4}, 150 \mathrm{MHz}$ ) spectrum of arbophyllinine B (3) 8

7 Figure S7. X-ray crystal structure of arbophyllidine (1)

$8 \quad$ Table S8. Crystal data and structure refinement parameters of arbophyllidine (1) $\quad 10$

9 Figure S9. X-ray crystal structure of arbophyllinine A (2) 11

10 Table S10. Crystal data and structure refinement parameters of arbophyllinine A (2) 12

11 Figure S11. Bijvoet-pair analysis of crystallographic data for arbophyllinine A (2), 13 generated from the software PLATON.

12 Figure S12. X-ray crystal structure of arbophyllinine B (3)

13 Table S13. Crystal data and structure refinement parameters of arbophyllinine B (3) 15

14 Table S14. DFT B3LYP/6-31G(d) atomic Cartesian coordinates ( $)$ for (-)-1 (5R, 16 $15 S)$ 


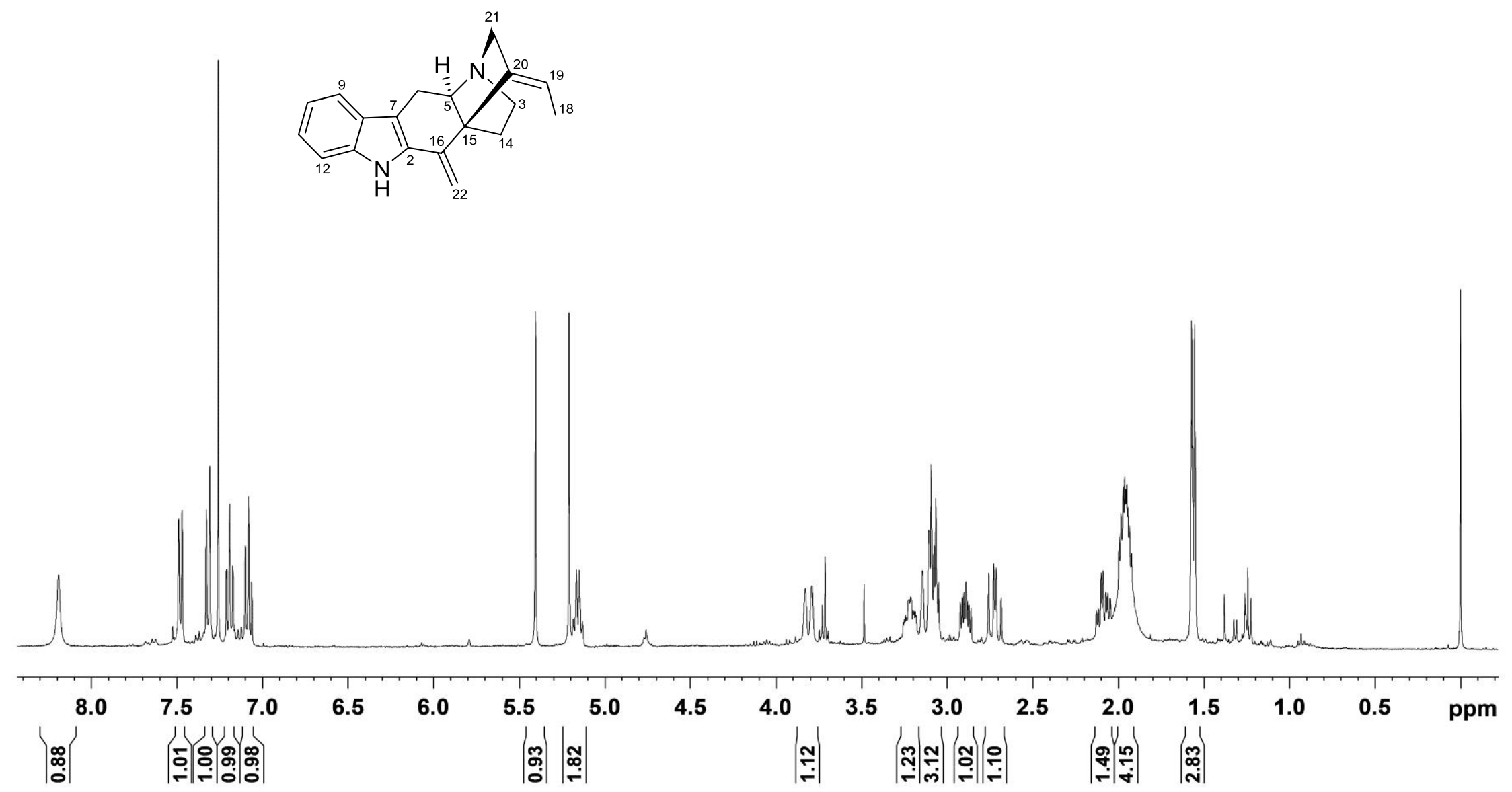

Figure S1. ${ }^{1} \mathrm{H}$ NMR $\left(\mathrm{CDCl}_{3}, 400 \mathrm{MHz}\right)$ spectrum of arbophyllidine (1) 

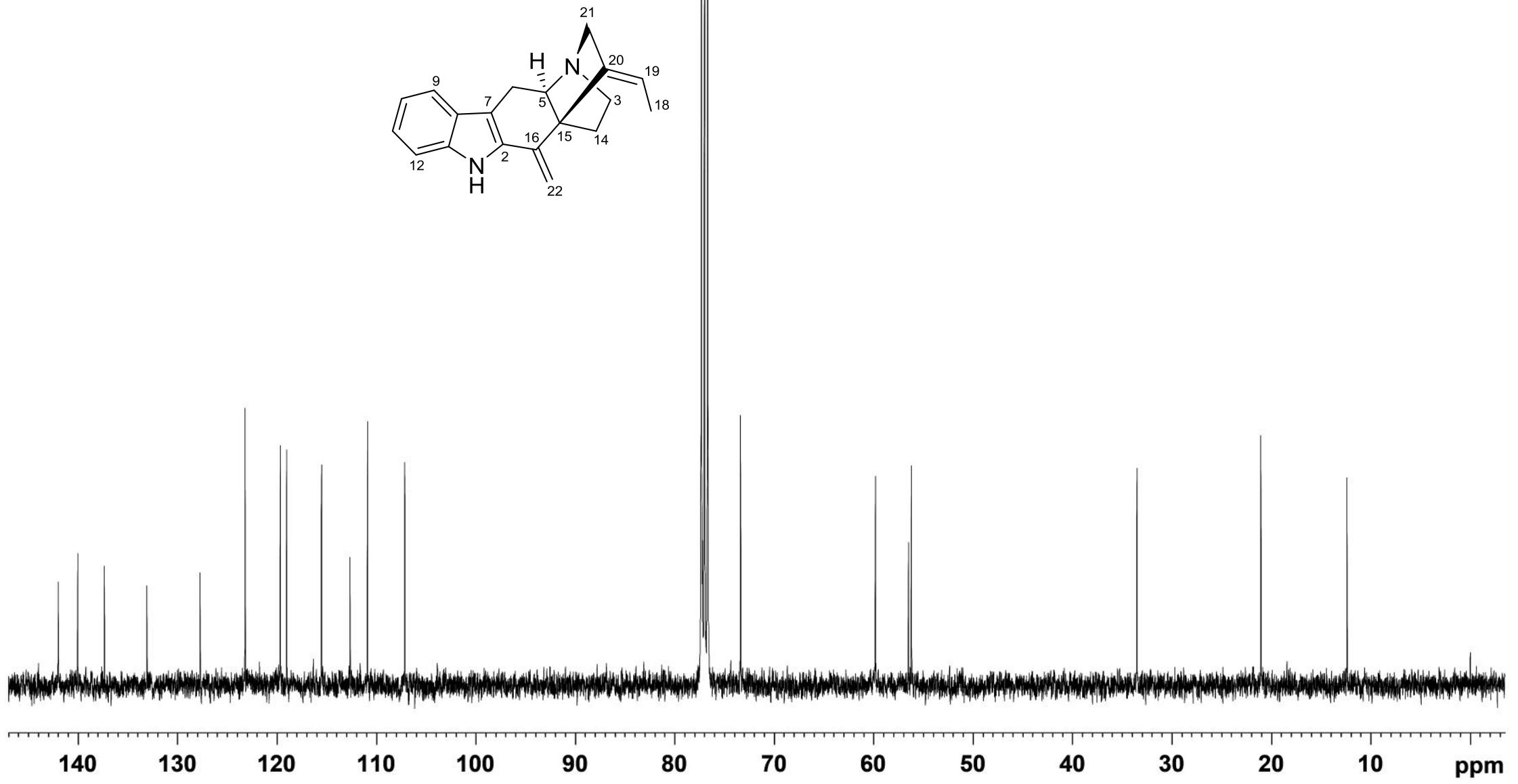

Figure S2. ${ }^{13} \mathrm{C}$ NMR $\left(\mathrm{CDCl}_{3}, 100 \mathrm{MHz}\right)$ spectrum of arbophyllidine (1) 


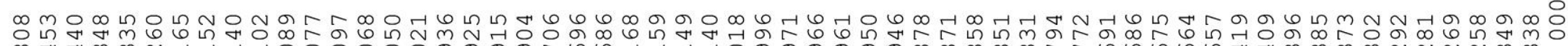
$\infty$ न $m$ m N

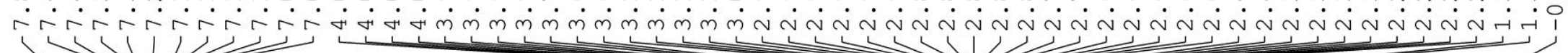

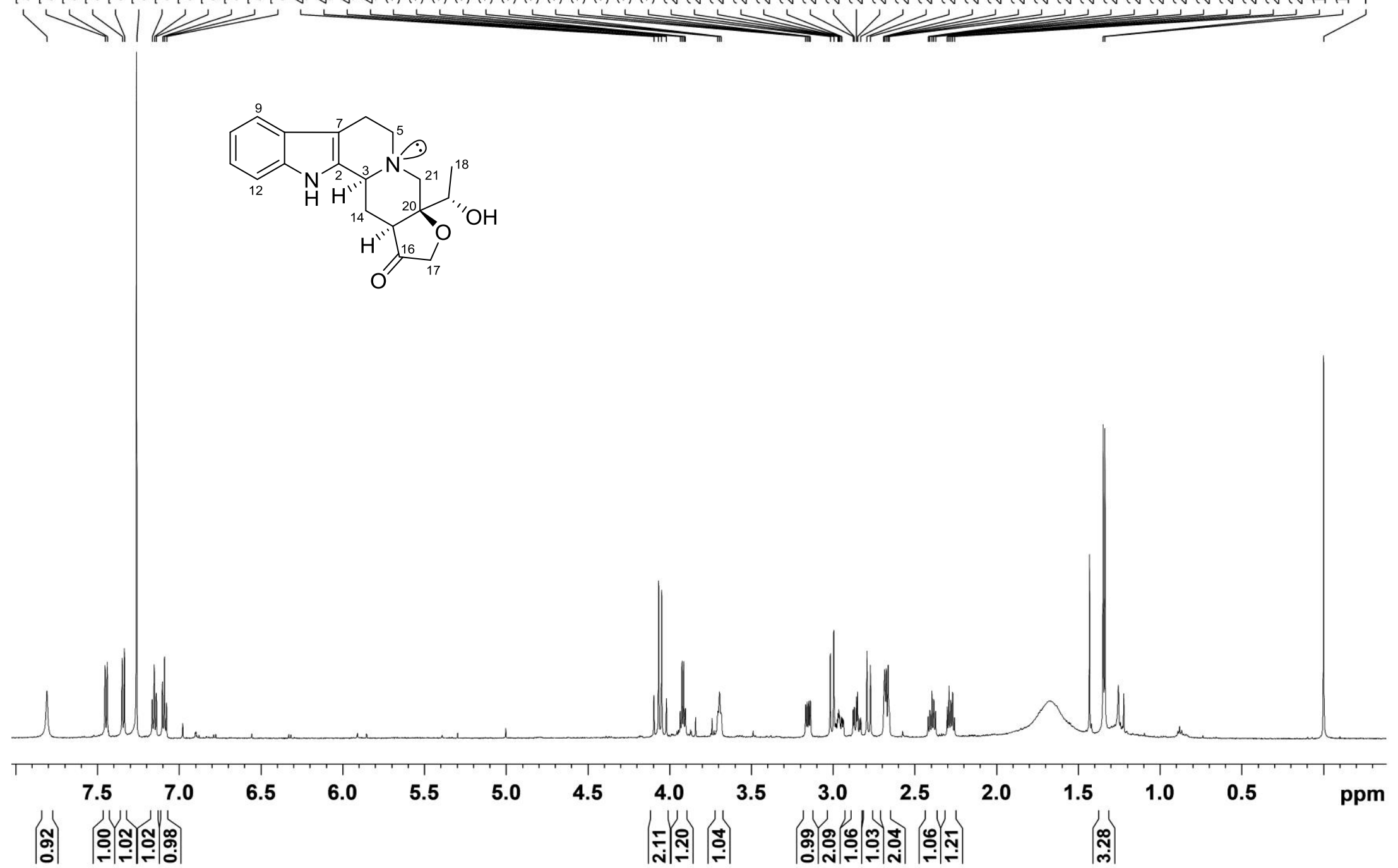

Figure S3. ${ }^{1} \mathrm{H}$ NMR $\left(\mathrm{CDCl}_{3}, 600 \mathrm{MHz}\right)$ spectrum of arbophyllinine A (2) 

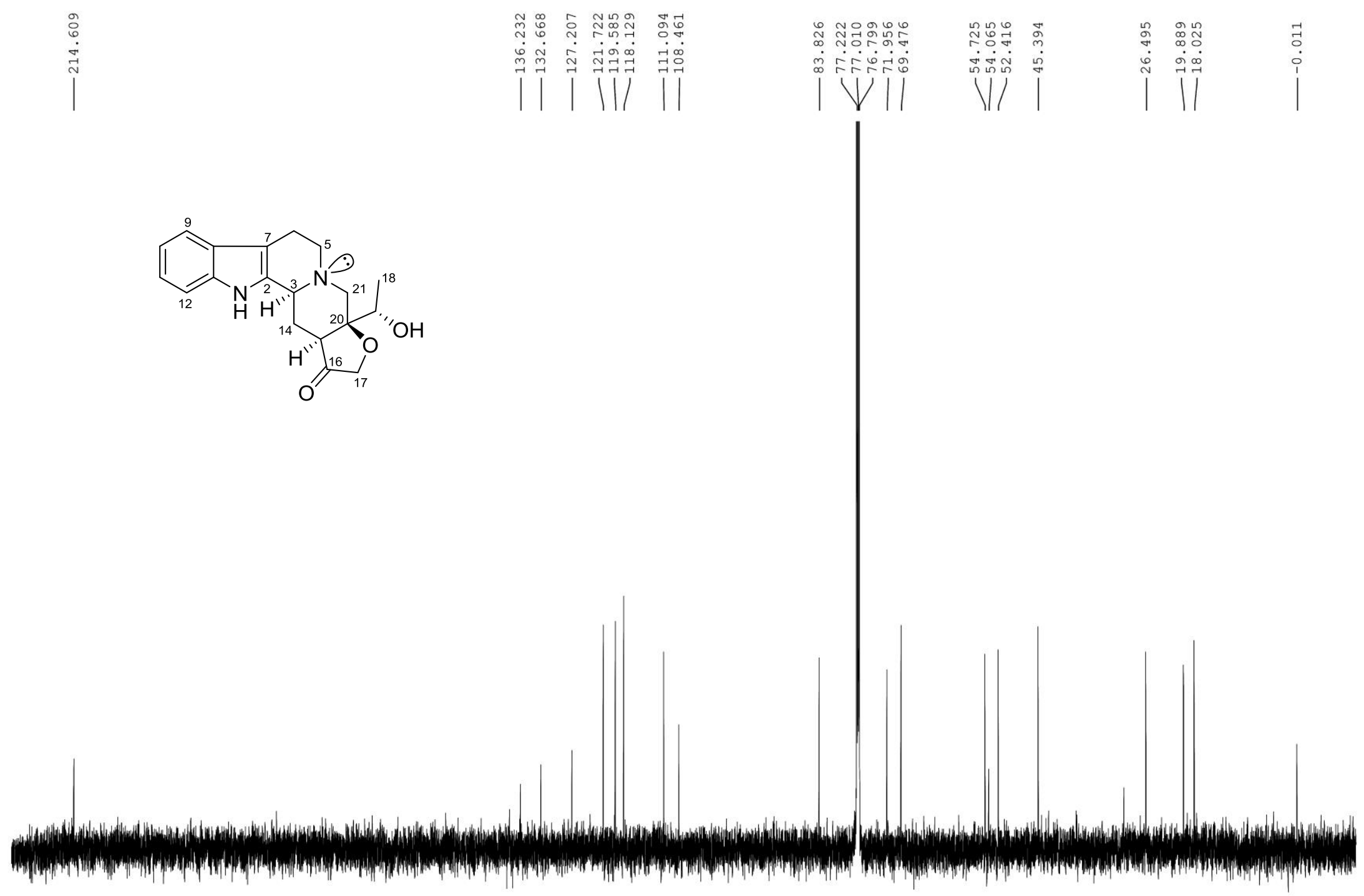

$\begin{array}{lllllllllllllllllllllll}220 & 210 & 200 & 190 & 180 & 170 & 160 & 150 & 140 & 130 & 120 & 110 & 100 & 90 & 80 & 70 & 60 & 50 & 40 & 30 & 20 & 10\end{array}$

ppm

Figure S4. ${ }^{13} \mathrm{C}$ NMR $\left(\mathrm{CDCl}_{3}, 150 \mathrm{MHz}\right)$ spectrum of arbophyllinine A (2) 


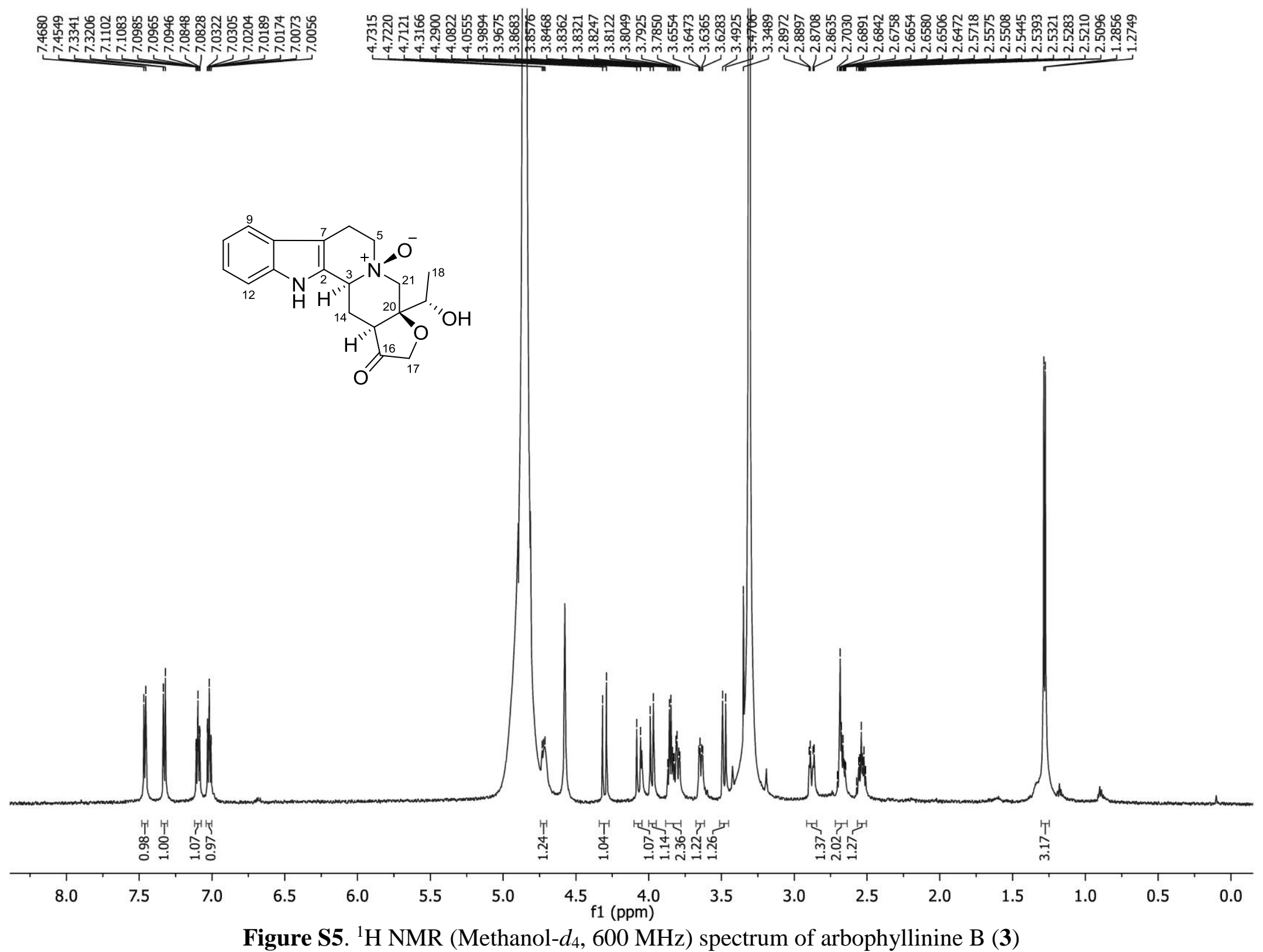




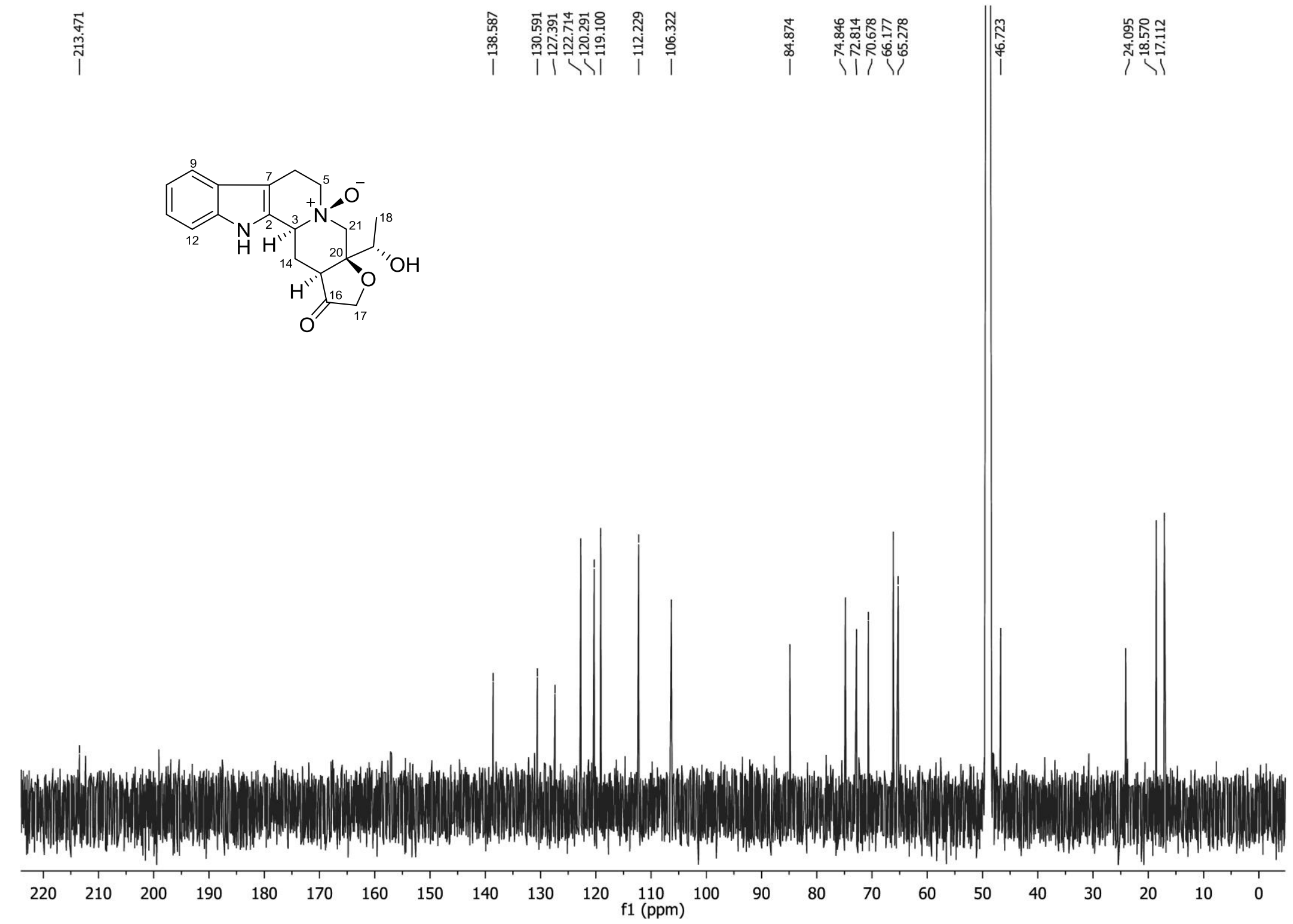

Figure S6. ${ }^{13} \mathrm{C}$ NMR (Methanol- $d_{4}, 150 \mathrm{MHz}$ ) spectrum of arbophyllinine B (3) 


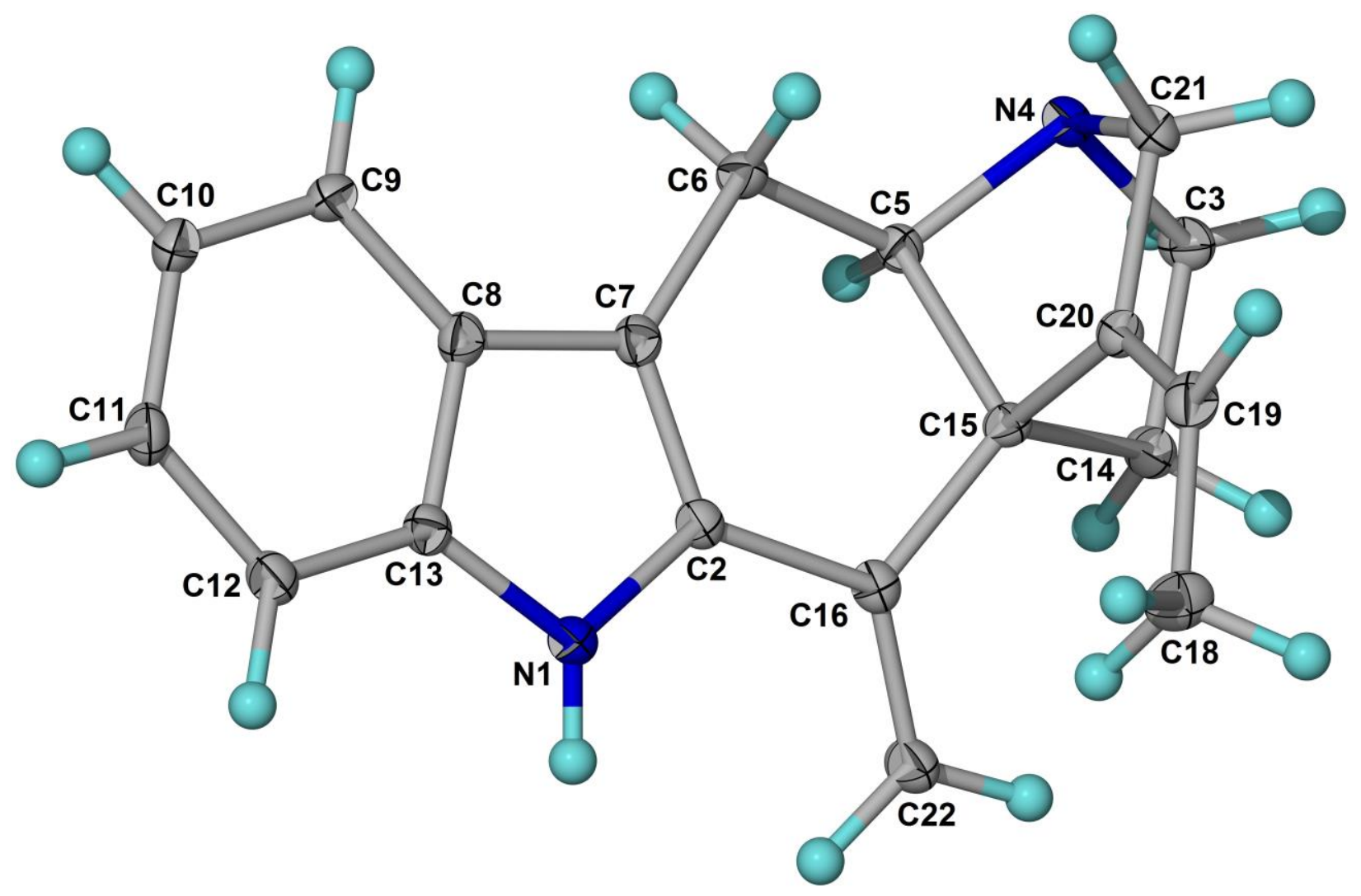

Figure S7. X-ray crystal structure of arbophyllidine (1) 
Table S8. Crystal data and structure refinement parameters of arbophyllidine (1)

\begin{tabular}{|c|c|}
\hline Empirical formula & $\mathrm{C}_{19} \mathrm{H}_{20} \mathrm{~N}_{2}$ \\
\hline Molecular formula & $\mathrm{C}_{19} \mathrm{H}_{20} \mathrm{~N}_{2}$ \\
\hline Molecular weight, $M_{r}$ & 276.37 \\
\hline Melting point & $>230{ }^{\circ} \mathrm{C} \mathrm{dec}$ \\
\hline Temperature during diffraction experiment, $T$ & $100 \mathrm{~K}$ \\
\hline X-ray source & Mo $K_{\alpha}$ \\
\hline Crystal system & Triclinic \\
\hline Space group & $P 1$ \\
\hline$a$ & $6.5325(3) \AA$ \\
\hline$b$ & $7.8576(5) \AA$ \\
\hline$c$ & $8.6225(5) \AA$ \\
\hline$\alpha$ & $109.428(6)^{\circ}$ \\
\hline$\beta$ & $94.781(4)^{\circ}$ \\
\hline$\gamma$ & $113.321(5)^{\circ}$ \\
\hline Volume, $V$ & $371.29(4) \AA^{3}$ \\
\hline No. of molecule per unit cell, $Z$ & 1 \\
\hline Density (calcd) & $1.236 \mathrm{mg} / \mathrm{mm}^{3}$ \\
\hline$F(000)$ & 148 \\
\hline Crystal size & $0.2 \times 0.12 \times 0.1 \mathrm{~mm}^{3}$ \\
\hline $2 \theta$ range for data collection & 6.16 to $60.152^{\circ}$ \\
\hline Index ranges & $-7 \leq h \leq 9,-10 \leq k \leq 9,-12 \leq l \leq 11$ \\
\hline Reflections collected & 3477 \\
\hline Independent reflections & $2558\left[R_{\text {int }}=0.0200, R_{\text {sigma }}=0.0409\right]$ \\
\hline Data/restraints/parameters & $2558 / 3 / 195$ \\
\hline Goodness-of-fit on $F^{2}$ & 1.058 \\
\hline Final $\mathrm{R}$ indexes $[I \geq 2 \sigma(I)]$ & $R_{1}=0.0427, w R_{2}=0.1052$ \\
\hline Final $\mathrm{R}$ indexes [all data] & $R_{1}=0.0471, w R_{2}=0.1102$ \\
\hline Largest diff. peak/hole / e $\AA^{-3}$ & $0.28 /-0.25$ \\
\hline Flack parameter & $-4.4(10)$ \\
\hline CCDC No.: & 1903211 \\
\hline
\end{tabular}




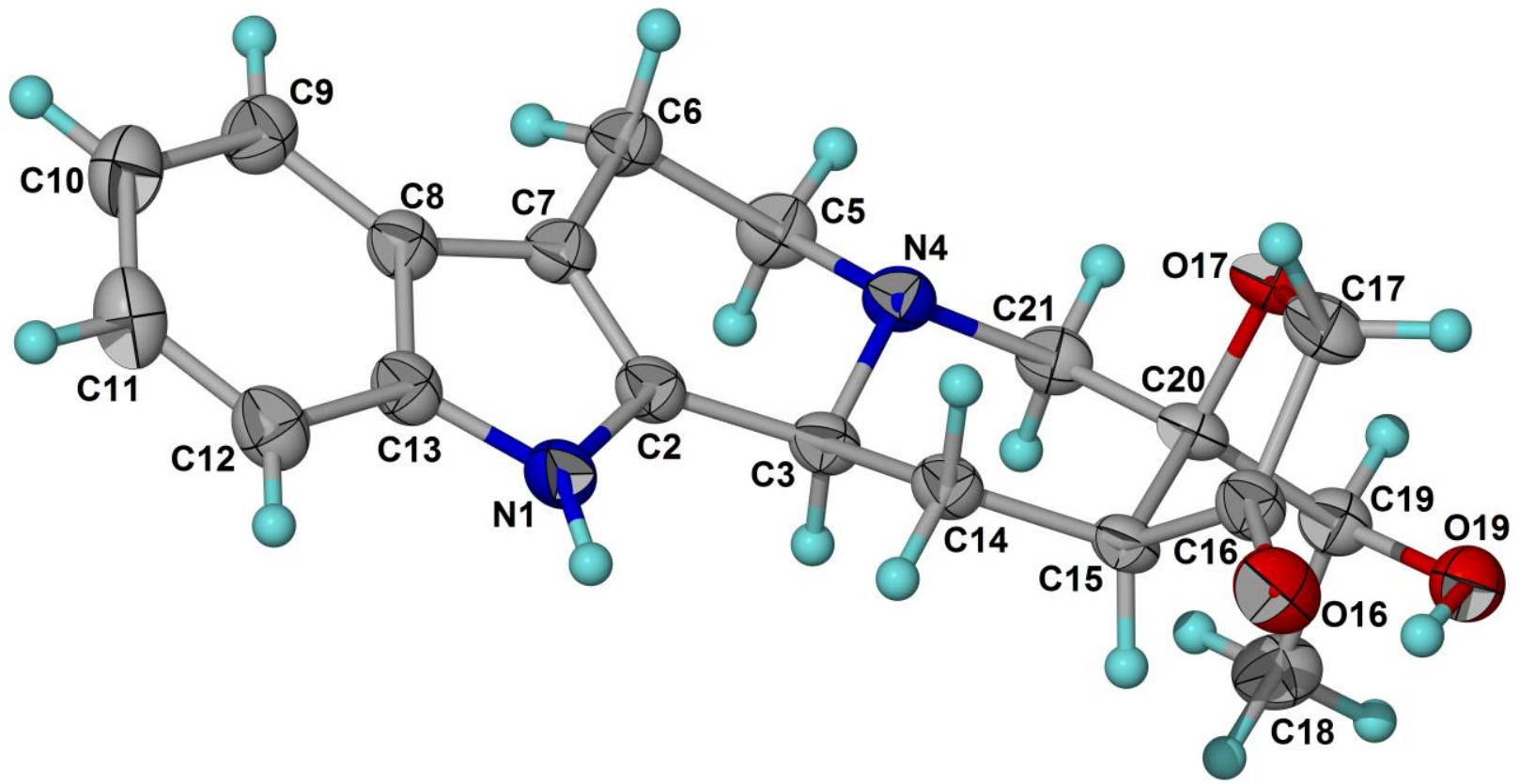

Figure S9. X-ray crystal structure of arbophyllinine A (2) 
Table S10. Crystal data and structure refinement parameters of arbophyllinine A (2)

\begin{tabular}{|c|c|}
\hline Empirical formula & $\mathrm{C}_{19} \mathrm{H}_{22} \mathrm{~N}_{2} \mathrm{O}_{3}$ \\
\hline Molecular formula & $>135$ oC dec. \\
\hline Molecular weight, $M_{r}$ & 326.38 \\
\hline Melting point & $>135^{\circ} \mathrm{C}$ dec. \\
\hline Temperature during diffraction experiment, $T$ & 293(2) K \\
\hline X-ray source & $\mathrm{Cu} K_{\alpha}$ \\
\hline Crystal system & Monoclinic \\
\hline Space group & $P 2_{1}$ \\
\hline$a$ & $10.1058(4) \AA$ \\
\hline$b$ & $5.4876(2) \AA$ \\
\hline$c$ & $14.6707(5) \AA$ \\
\hline$\alpha$ & $90^{\circ}$ \\
\hline$\beta$ & $91.494(3)^{\circ}$ \\
\hline$\gamma$ & $90^{\circ}$ \\
\hline Volume, $V$ & $813.31(5) \AA^{3}$ \\
\hline No. of molecule per unit cell, $Z$ & 2 \\
\hline Density (calcd) & $1.333 \mathrm{mg} / \mathrm{mm}^{3}$ \\
\hline$F(000)$ & 348 \\
\hline Crystal size & $0.15 \times 0.1 \times 0.1 \mathrm{~mm}^{3}$ \\
\hline $2 \theta$ range for data collection & 8.754 to $148.512^{\circ}$ \\
\hline Index ranges & $-12 \leq h \leq 12,-6 \leq k \leq 6,-18 \leq l \leq 18$ \\
\hline Reflections collected & 12849 \\
\hline Independent reflections & $3199\left[R_{\text {int }}=0.0210, R_{\text {sigma }}=0.0144\right]$ \\
\hline Data/restraints/parameters & $3199 / 1 / 223$ \\
\hline Goodness-of-fit on $F^{2}$ & 1.047 \\
\hline Final $\mathrm{R}$ indexes $[I \geq 2 \sigma(I)]$ & $R_{1}=0.0328, w R_{2}=0.0907$ \\
\hline Final $\mathrm{R}$ indexes [all data] & $R_{1}=0.0342, w R_{2}=0.0923$ \\
\hline Largest diff. peak/hole / e $\AA^{-3}$ & $0.21 /-0.16$ \\
\hline Flack parameter, $x$ & $-0.02(8)$ \\
\hline Hooft parameter, $y$ & $-0.03(7)$ \\
\hline Parsons parameter, $z$ & $-0.01(7)$ \\
\hline CCDC No.: & 1903212 \\
\hline
\end{tabular}




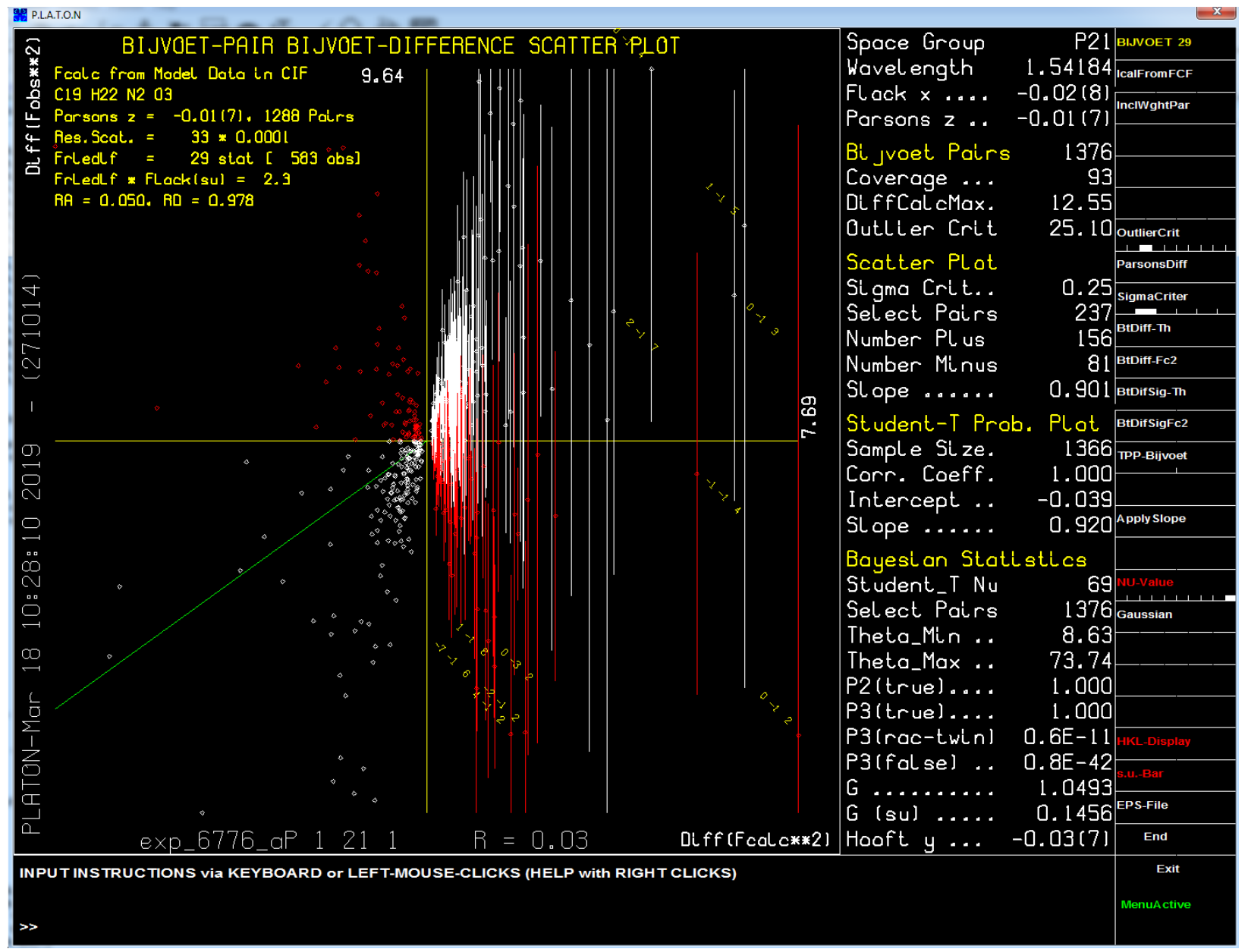

Figure S11. Bijvoet-pair analysis of crystallographic data for arbophyllinine A (2), generated from the software PLATON. 


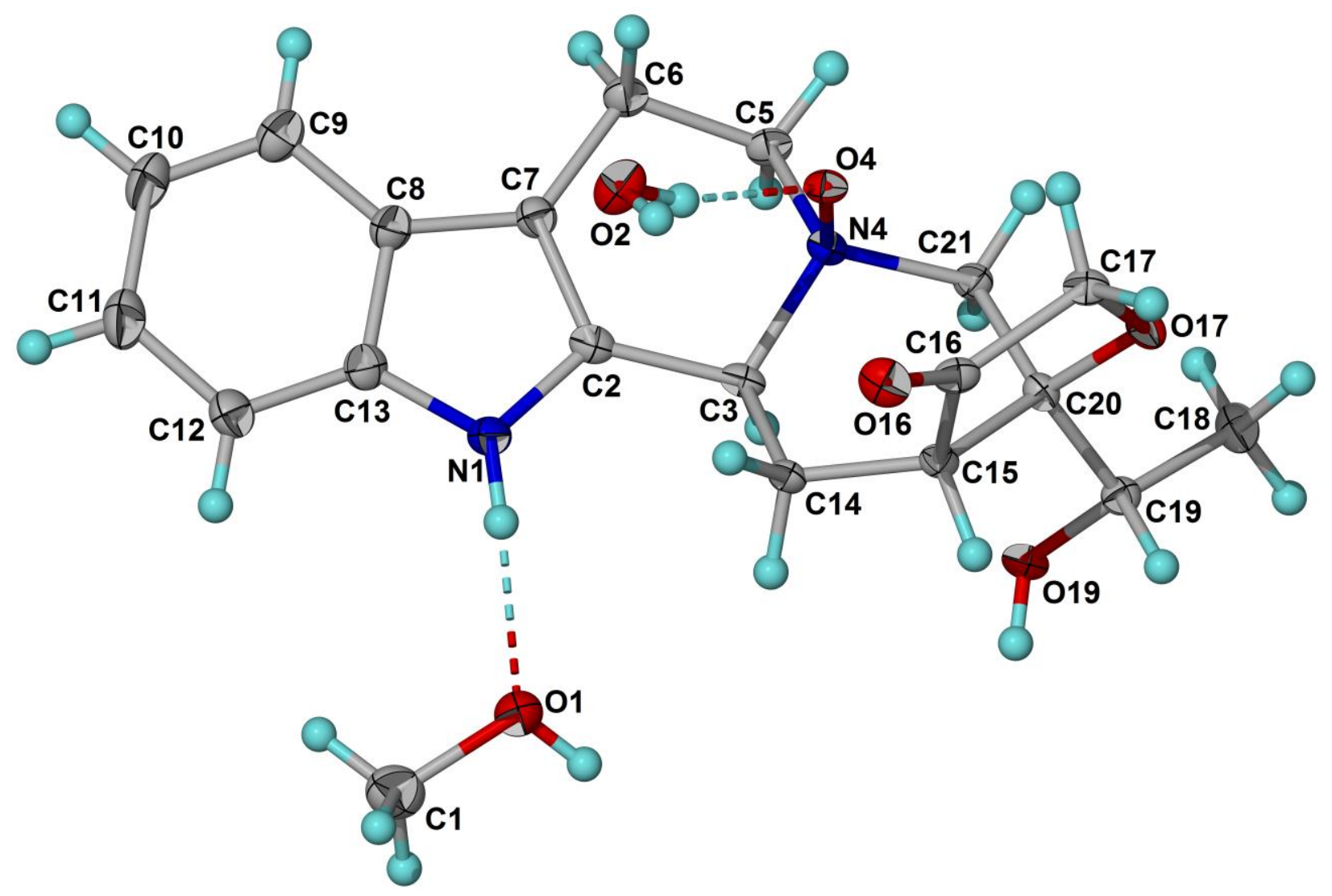

Figure S12. X-ray crystal structure of arbophyllinine B (3) 
Table S13. Crystal data and structure refinement parameters of arbophyllinine B (3)

\begin{tabular}{|c|c|}
\hline Empirical formula & $\mathrm{C}_{20} \mathrm{H}_{28} \mathrm{~N}_{2} \mathrm{O}_{6}$ \\
\hline Molecular formula & $\mathrm{C}_{19} \mathrm{H}_{22} \mathrm{~N}_{2} \mathrm{O}_{4} \cdot \mathrm{CH}_{3} \mathrm{OH} \cdot \mathrm{H}_{2} \mathrm{O}$ \\
\hline Molecular weight, $M_{r}$ & 392.44 \\
\hline Melting point & $>215^{\circ} \mathrm{C} \mathrm{dec}$ \\
\hline Temperature during diffraction experiment, $T$ & $100(2) \mathrm{K}$ \\
\hline X-ray source & Mo $K_{\alpha}$ \\
\hline Crystal system & Monoclinic \\
\hline Space group & $P 2_{1}$ \\
\hline$a$ & $6.7413(5) \AA$ \\
\hline$b$ & $41.936(4) \AA$ \\
\hline$c$ & $6.7867(5) \AA$ \\
\hline$\alpha$ & $90^{\circ}$ \\
\hline$\beta$ & $101.551(7)^{\circ}$ \\
\hline$\gamma$ & $90^{\circ}$ \\
\hline Volume, $V$ & $1879.8(3) \AA^{3}$ \\
\hline No. of molecule per unit cell, $Z$ & 4 \\
\hline Density (calcd) & $1.387 \mathrm{mg} / \mathrm{mm}^{3}$ \\
\hline$F(000)$ & 840 \\
\hline Crystal size & $0.5 \times 0.4 \times 0.4 \mathrm{~mm}^{3}$ \\
\hline $2 \theta$ range for data collection & 5.828 to $60.608^{\circ}$ \\
\hline Index ranges & $-8 \leq h \leq 9,-24 \leq k \leq 54,-6 \leq l \leq 8$ \\
\hline Reflections collected & 9424 \\
\hline Independent reflections & $7210\left[R_{\text {int }}=0.0298, R_{\text {sigma }}=0.0677\right]$ \\
\hline Data/restraints/parameters & $7210 / 1 / 519$ \\
\hline Goodness-of-fit on $F^{2}$ & 1.084 \\
\hline Final $\mathrm{R}$ indexes $[I \geq 2 \sigma(I)]$ & $R_{1}=0.0546, w R_{2}=0.1214$ \\
\hline Final $\mathrm{R}$ indexes [all data] & $R_{1}=0.0695, w R_{2}=0.1337$ \\
\hline Largest diff. peak/hole / e $\AA^{-3}$ & $0.29 /-0.44$ \\
\hline CCDC No.: & 1903213 \\
\hline
\end{tabular}


Table S14. DFT B3LYP/6-31G(d) atomic Cartesian coordinates $(\AA)$ for compound (-)-1 (5R, $15 S$ )

\begin{tabular}{|c|c|c|c|}
\hline Atom & $X$ & $Y$ & $\mathrm{Z}$ \\
\hline $\mathrm{C}$ & 5.072807 & -0.027527 & 0.179690 \\
\hline $\mathrm{C}$ & 4.198338 & 0.974136 & -0.228044 \\
\hline $\mathrm{C}$ & 2.838093 & 0.657416 & -0.294659 \\
\hline $\mathrm{C}$ & 2.343658 & -0.635009 & 0.044848 \\
\hline $\mathrm{C}$ & 3.254814 & -1.627601 & 0.447785 \\
\hline $\mathrm{C}$ & 4.606789 & -1.316593 & 0.512036 \\
\hline $\mathrm{H}$ & 6.136206 & 0.187373 & 0.240828 \\
\hline $\mathrm{H}$ & 4.561132 & 1.965501 & -0.487286 \\
\hline $\mathrm{H}$ & 2.904668 & -2.623286 & 0.707901 \\
\hline $\mathrm{H}$ & 5.319781 & -2.075028 & 0.823513 \\
\hline $\mathrm{C}$ & 0.919113 & -0.595767 & -0.124043 \\
\hline $\mathrm{C}$ & 0.593661 & 0.678435 & -0.551986 \\
\hline $\mathrm{N}$ & 1.757993 & 1.427701 & -0.670074 \\
\hline $\mathrm{H}$ & 1.787160 & 2.417743 & -0.856246 \\
\hline $\mathrm{C}$ & -0.098150 & -1.679491 & 0.099441 \\
\hline $\mathrm{H}$ & 0.264526 & -2.646870 & -0.270717 \\
\hline $\mathrm{H}$ & -0.271164 & -1.812303 & 1.174925 \\
\hline $\mathrm{C}$ & -1.396939 & -1.305634 & -0.624076 \\
\hline $\mathrm{C}$ & -1.827603 & 0.198034 & -0.445187 \\
\hline $\mathrm{C}$ & -0.744456 & 1.156655 & -0.883510 \\
\hline $\mathrm{C}$ & -0.979731 & 2.285181 & -1.578106 \\
\hline $\mathrm{H}$ & -1.988100 & 2.609877 & -1.810821 \\
\hline $\mathrm{H}$ & -0.176638 & 2.917933 & -1.947726 \\
\hline $\mathrm{C}$ & -3.146432 & 0.174905 & -1.270710 \\
\hline
\end{tabular}




\begin{tabular}{llll}
$\mathrm{C}$ & -3.635273 & -1.300191 & -1.047598 \\
$\mathrm{H}$ & -4.636487 & -1.365500 & -0.613376 \\
$\mathrm{H}$ & -3.643653 & -1.857783 & -1.989533 \\
$\mathrm{~N}$ & -2.645994 & -1.933176 & -0.133774 \\
$\mathrm{C}$ & -2.260308 & 0.146609 & 1.026556 \\
$\mathrm{C}$ & -2.818091 & -1.281358 & 1.188713 \\
$\mathrm{H}$ & -2.270656 & -1.844993 & 1.952589 \\
$\mathrm{H}$ & -3.875150 & -1.300283 & 1.474634 \\
$\mathrm{C}$ & -2.232262 & 1.038339 & 2.021292 \\
$\mathrm{H}$ & -2.626607 & 0.694523 & 2.981655 \\
$\mathrm{C}$ & -1.742453 & 2.459427 & 2.031763 \\
$\mathrm{H}$ & -2.548999 & 3.140170 & 2.337989 \\
$\mathrm{H}$ & -0.935480 & 2.585396 & 2.766975 \\
$\mathrm{H}$ & -1.373391 & 2.786547 & 1.059363 \\
$\mathrm{H}$ & -3.868333 & 0.919835 & -0.923716 \\
$\mathrm{H}$ & -2.938093 & 0.370843 & -2.328363 \\
$\mathrm{H}$ & -1.278173 & -1.531163 & -1.690879 \\
\hline & & & \\
\hline & & & \\
\hline
\end{tabular}

